\title{
Recollection and Familiarity Make Independent Contributions to Memory Judgments
}

\author{
Lisa H. Evans and Edward L. Wilding \\ Wales Institute of Cognitive Neuroscience, Cardiff University Brain Research Imaging Centre, School of Psychology, Cardiff University, Cardiff, CF10 3AT, \\ Wales, United Kingdom
}

Recognition memory can be supported by the processes of recollection and familiarity. Recollection is recovery of qualitative information about a prior event. Familiarity is a scalar strength signal that permits judgments of prior occurrence. There is vigorous debate about how these processes are conceptualized, how they contribute to memory judgments, and which brain regions support them. One popular method for investigating these questions is the Remember/Know procedure, where subjects give a Remember response to studied stimuli for which they can recover contextual details of the study encounter, and a Know response when details are not recovered but subjects nevertheless believe that a stimulus was studied. According to one model, Remember responses are strong memories that are typically associated with relatively high levels of recollection and familiarity. Know responses are weaker memories and are typically associated with lower levels of both processes. Data inconsistent with this account were obtained in this experiment, where magnetoencephalographic (MEG) measures of neural activity were acquired in the test phase of a verbal memory task where healthy human volunteers made Remember, Know, or New judgments to studied and unstudied words. An MEG index of the process of recollection was larger for Remember than Know judgments, whereas the reverse was true for a MEG index of familiarity. Critically, this result is predicted by a model where recollection and familiarity make independent contributions to Remember and Know judgments, and provides a powerful constraint when mapping memory processes onto their neural substrates.

\section{Introduction}

How recognition memory is supported is debated vigorously. While there is some consensus that the processes of recollection and familiarity contribute, the questions of how they do so and how the medial temporal lobe supports these processes remain contentious (Eichenbaum et al., 2007; Squire et al., 2007; Wixted, 2007; Yonelinas and Parks, 2007). Recollection is recovery of qualitative information about an encoded event. Familiarity is a graded strength signal that can support judgments of prior occurrence, and one widely used task that has been used to study these processes is the Remember/Know procedure (Tulving, 1985; Gardiner and Java, 1993).

In this procedure, a Remember response is to be given when memory of an event includes specific details. A Know response is to be given when specific details are not recovered but people believe that the event occurred (Rajaram, 1993). According to a widely recognized dual-process recognition memory model (Yonelinas, 1994, 2002), recollection supports Remember judgments, while familiarity in the absence of recollection supports Know judgments. These two processes are considered to be inde-

Received Dec. 22, 2011; revised March 28, 2012; accepted April 5, 2012.

Author contributions: L.H.E. and E.L.W. designed research; L.H.E. performed research; L.H.E. and E.L.W. analyzed data; L.H.E. and E.L.W. wrote the paper.

This work was supported by the UK Biotechnology and Biological Sciences Research Council and the Wales Institute of Cognitive Neuroscience.

Correspondence should be addressed to Prof. E. Wilding, School of Psychology, Cardiff University, Cardiff, CF10 3AT.E-mail:wildinge@cardiff.ac.uk.

DOI:10.1523/JNEUROSCI.6396-11.2012

Copyright $\odot 2012$ the authors $\quad 0270-6474 / 12 / 327253-05 \$ 15.00 / 0$ pendent. By another view, under typical task instructions, Remember/Know judgments separate strong from weak memories (Donaldson, 1996; Wixted, 2007, 2009; Wixted and Mickes, 2010). In one influential account, the distinction is made by assessing a sum of recollection and familiarity strengths (Wixted, 2007). Remember responses are strong memories, which are associated on average with relatively high levels of recollection and familiarity. Know responses are weak memories, which are associated on average with lower levels of both processes (Wixted, 2007, 2009).

In this experiment, magnetoencephalographic (MEG) indices of recollection and familiarity were used to adjudicate between these accounts (Bridson et al., 2009). If the memory strength account is accurate, Remember responses will elicit larger indices of recollection and familiarity than will Know responses. The independent dual-process model described above also predicts larger indices of recollection for Remember than Know responses. Critically, however, it predicts the opposite profile for the index of familiarity - it will be larger for Know than for Remember responses. This is because if the two processes are independent, there is no lower limit for the level of familiarity associated with Remember responses, whereas there is a limit that must be exceeded for Know responses to be given (for the same observation in related terms, see Yu and Rugg, 2010, Berry et al., 2012). Hence, if familiarity is indexed by a measure of neural activity averaged over many trials, the index will be larger for Know than for Remember responses.

To test these accounts, event-related fields (ERFs) were recorded for memory judgments in the test phase of a Remem- 
ber/Know task. ERFs are segments of the on-going MEG record of neural activity. At issue is the accuracy of models with different relationships between the processes of recollection and familiarity.

\section{Materials and Methods}

Participants were 21 right-handed, healthy native English speakers. All gave written consent, and the experiment was approved by the Cardiff University School of Psychology Ethics Committee. The analyses reported here are based on data from 17 participants ( 14 females; mean age, 21 years). Four were excluded because they contributed $<14$ trials to at least one of the critical experiment conditions: two because of large ocular artifacts and two because of excessive magnetic interference during data acquisition.

There were four study test blocks. Each study block contained 48 English words (4-9 letters; 1-7 occurrences per million). A cue preceded each word. The "CONCRETE/ABSTRACT?" cue directed participants to assess whether the word referred to a concrete or abstract entity. The "ALPHABETIC?" cue directed participants to assess whether the first and last letters of the word were in alphabetic order. Responses were made on a key pad with the left and right index fingers. The two cues appeared equally often in a random order. Each study trial consisted of the cue (300 $\mathrm{ms})$, a blank screen $(1000 \mathrm{~ms})$, the study word $(300 \mathrm{~ms})$, a blank screen for the time taken for the study response plus $1000 \mathrm{~ms}$, a "BLINK NOW" instruction (1000 ms), and a blank screen (1000 ms).

All words presented in study blocks were represented in the immediately following test block, intermixed randomly with 48 unstudied words. No words were repeated across blocks. Each test trial comprised a fixation asterisk (1000 ms), a blank screen $(200 \mathrm{~ms})$, the test word $(300 \mathrm{~ms})$, a blank screen that remained for the period of time taken for a response plus $1000 \mathrm{~ms}$, a "BLINK NOW" instruction (1000 $\mathrm{ms})$, and a blank screen (1000 ms). Participants were asked to make a Remember, Know, or New response to each word. The explanation for these terms was based upon the instructions detailed by Rajaram (1993), and was supplemented with further examples of the Remember/Know distinction.

Participants were asked to balance accuracy and speed of responding, to minimize head movement, and to blink only when the "BLINK NOW" instruction was visible. Responses at test were made with the index and middle fingers of the left and right hands. Remember and Know responses were made on the same hand, New responses on the other. Hands used at study and test were balanced across participants. Four complete task lists were created, ensuring that, across lists, all words were presented as old and new stimuli at test, and that all old words were encoded under both study conditions.

Whole-head MEG recordings were made using a 275-channel radial gradiometer system (VSM MedTech). An additional 29 reference channels were recorded and the primary sensors were analyzed as synthetic third-order gradiometers (Vrba and Robinson, 2001). Three of the 275 channels were removed due to excessive sensor noise. The sampling rate was $300 \mathrm{~Hz}$ and recordings were bandpass filtered off-line $(0.03-40 \mathrm{~Hz})$. Intraindividual head movement was kept to a minimum, and head position was localized at the start and end of the experiment. ERFs were formed for $2100 \mathrm{~ms}$ epochs, including a $100 \mathrm{~ms}$ prestimulus baseline relative to which all mean signal strengths were computed. Trials containing large signal and/or EOG artifacts were rejected before averaging.

Averaged ERFs were formed for each participant for Remember and Know responses to old words that had received an alphabetic (vowel order) judgment at study (the shallow encoding task), Remember responses to old words that had received a concrete/abstract study judgment (the deep task), and new responses to new words (correct rejections). The analyses did not include averaged ERFs for correct Know responses to test words encountered in the deep encoding condition as only 12 participants contributed 14 or more artifact-free trials to this response category. ERFs associated with misses (new responses to old words) were formed by collapsing across words from the deep and shallow encoding tasks. The mean numbers of trials in each response category were as follows: Remember (shallow) $=24$ (range, 14-42), Know
Table 1. Probabilities of Remember, Know, and New responses to new (unstudied) test words and to old words separated according to encoding task (deep/shallow)

\begin{tabular}{llll}
\hline & \multicolumn{2}{l}{ Word status } & \\
\cline { 2 - 3 } & Old & New \\
\cline { 2 - 4 } Response & Deep & Shallow & $0.02(0.02)$ \\
\hline Remember & $0.60(0.18)$ & $0.30(0.11)$ & $1070(280)$ \\
RT (ms) & $1123(285)$ & $1093(262)$ & $0.13(0.06)$ \\
Know & $0.25(0.12)$ & $0.35(0.09)$ & $1435(372)$ \\
RT (ms) & $1372(346)$ & $1352(290)$ & $0.85(0.08)$ \\
New & $0.14(0.10)$ & $0.35(0.14)$ & $982(196)$ \\
RT (ms) & $1018(202)$ & $1110(269)$ & \\
\hline
\end{tabular}

Standard deviations in parentheses.

$($ shallow $)=28(14-40)$, Correct Rejection $=130(74-162)$, Remember $($ deep $)=51(26-80)$, Miss (deep and shallow $)=37(16-72)$. Across participants, an average of $72 \%$ of the trials in the combined Miss category were words encountered in the shallow encoding condition.

The ERFs recorded from adjacent sensors were averaged, forming 12 clusters in a $6 \times 2$ array above frontal and posterior scalp over the left and right hemispheres. The terms inferior, midlateral, and superior are used below to denote the distances between sensor clusters and the midline. Data points in each cluster are the average signal from six MEG sensors (except for the left frontal inferior cluster, where one sensor was removed due to excessive noise). For a similar approach to data reduction for event-related potentials (ERPs), see Curran (1999, 2000); for ERFs, see Bridson et al. (2009). The Geisser-Greenhouse correction was applied to ANOVAs where necessary (Greenhouse and Geisser, 1959). Corrected degrees of freedom are reported in the text.

\section{Results}

Behavior

There was a trend for study phase reaction times (RTs) to be shorter for words in the deep task (1493 vs $1633 \mathrm{~ms} ; t_{(16)}=1.95$, $p=0.07)$. Mean proportions of Remember, Know, and New responses for new words and for old words split by task are shown in Table 1, along with the corresponding RTs. Old/new discrimination $\left(p_{\text {hit }}-p_{\text {false alarm }}\right.$ collapsed across Remember and Know judgments) was superior following deep encoding ( 0.70 vs 0.49 ; $\left.t_{(16)}=10.37, p<0.001\right)$. The probability of a Remember response to an old word was higher after deep than after shallow encoding $\left(t_{(16)}=9.92, p<0.001\right)$. The reverse was true for Know responses $\left(t_{(16)}=2.83, p<0.05\right)$. RTs were faster for correct responses to new words than for either Know or Remember responses to old words (minimum $t_{(16)}=2.22, p<0.05$ ). A $2 \times 2$ ANOVA on RTs to old words with factors of response category (Remember/Know) and encoding task (deep/shallow) revealed only that Remember responses were faster than Know responses $\left(F_{(1,16)}=17.47, p<0.01\right)$.

\section{Event-related fields}

The analyses were conducted over data for 300-500 and 500-800 $\mathrm{ms}$ poststimulus epochs. These were selected because neural indices of familiarity and recollection have been documented in these periods in ERF and ERP memory studies (Tendolkar et al., 2000; Rugg and Curran, 2007; Bridson et al., 2009). In their ERF study, in which the same MEG acquisition parameters were used, Bridson and colleagues (2009) identified a likely index of familiarity at sensors located above the anterior scalp between 300 and $500 \mathrm{~ms}$, and a likely index of recollection at sensor locations above the posterior scalp between 500 and 800 ms. Guided by these outcomes, the analyses for the 300-500 ms epoch in this experiment were restricted to clusters above frontal scalp loca- 
a
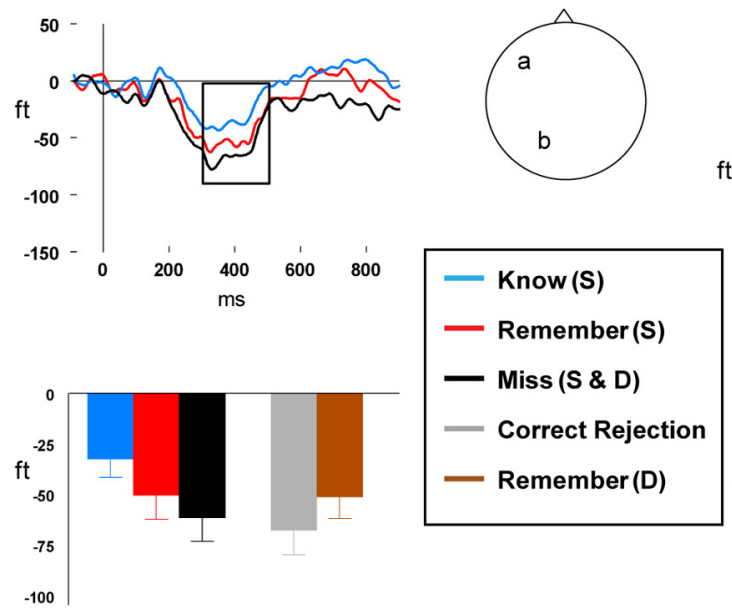

\section{b}
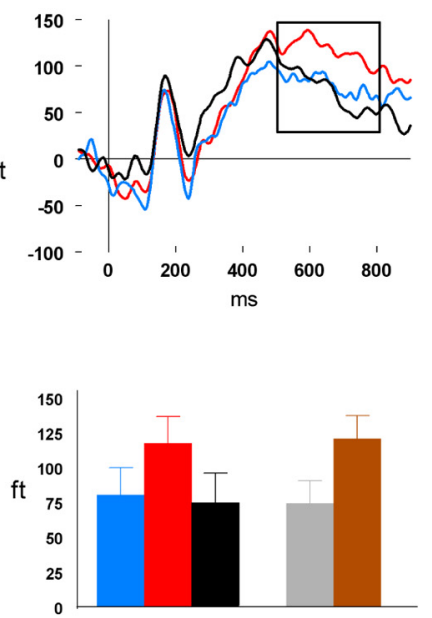

Figure 1. $\quad \boldsymbol{a}, \boldsymbol{b}$, Top, ERF waveforms averaged across a cluster of sensors at left anterior locations $(\boldsymbol{a})$ and left superior-posterior locations $(\boldsymbol{b})$. Bottom, Mean signal strength measures (in femtoteslas) for the 300-500 ms poststimulus period at left anterior locations $(\boldsymbol{a})$ and for the $500-800 \mathrm{~ms}$ period at left superior-posterior locations $(\boldsymbol{b})$.

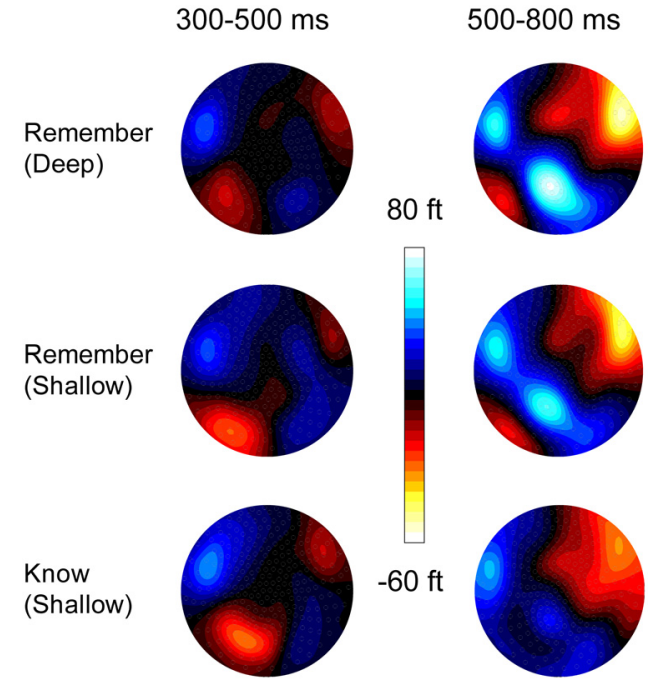

Figure 2. Scalp maps showing distributions of ERF activity for the 300-500 and 500-800 ms poststimulus epochs. The maps were computed based on values obtained by subtracting mean signal strengths associated with correct rejections from three kinds of Remember/Know response. Top, Remember responses to words subjected to deep encoding. Middle, Remember responses to words subjected to shallow encoding. Bottom, Know responses to words subjected to shallow encoding.

tions, and the analyses for the later epoch were restricted to clusters above posterior scalp locations.

In both epochs, an initial contrast was conducted between mean ERF signal strength measures for correct responses to new test words (correct rejections) and Remember responses to old test words that had been subjected to deep encoding. Each analysis included data from six clusters, three located over each hemisphere. The outcomes of these analyses comprised an independent means of identifying clusters in which subsequent analyses addressing the key experiment hypotheses were conducted. These subsequent analyses were restricted to ERFs associated with misses, and those for old words studied in the shallow encoding task that attracted either Remember or Know judgments (Fig. 1).

The initial ANOVA for the 300-500 ms epoch included the factors of response category (2 levels: correct rejection, remem- ber), hemisphere (2), and cluster location (inferior, midlateral, superior). The interaction between response category and hemisphere was significant $\left(F_{(1,16)}=8.08\right.$, $p<0.025)$, and separate analyses by hemisphere revealed a reliable effect of response category for clusters over the left hemisphere only $\left(F_{(1,16)}=10.28, p<\right.$ 0.01 ; Fig. 2). In the absence of an interaction involving cluster, the analyses conducted to determine the fit between the data and the predictions outlined in the Introduction comprised paired $t$ tests on mean signal strengths averaged over the three clusters above left-hemisphere frontal scalp. The mean signal strengths for these data points can be seen in Figure $1 a$. In keeping with the data shown in Figure 1, the ERF signal strengths in the 300-500 ms epoch for words attracting Know responses and subjected to shallow encoding were greater than those associated with Remember responses $\left(t_{(16)}=2.67\right.$, $p<0.05)$ and misses $\left(t_{(16)}=3.75, p<0.01\right)$. Strengths for the last two response categories did not differ significantly.

The initial analysis for the $500-800 \mathrm{~ms}$ epoch (factors as above) revealed an interaction between response category, hemisphere, and location $\left(F_{(1.3,20.7)}=5.84, p<0.025\right)$. An analysis restricted to clusters above the right hemisphere revealed no reliable effects involving response category, while the analysis for clusters above the left hemisphere revealed an interaction between category and cluster $\left(F_{(1.3,20.7)}=8.27, p<0.01\right)$, because the differences between these categories are largest for the superior cluster (Fig. 2). Paired $t$ tests taking measures from this cluster for the three critical response categories (Fig. $1 b$ ) revealed only that mean signal strengths associated with Remember responses for words subjected to shallow encoding were significantly greater than those associated with Know responses $\left(t_{(16)}=2.78, p<0.05\right)$ as well as misses $\left(t_{(16)}=3.20, p<0.01\right)$.

\section{Discussion}

ERF indices of the processes of familiarity and recollection were measured during the test phase of a Remember/Know task. ERF signal changes linked to familiarity were analyzed between 300 and $500 \mathrm{~ms}$ poststimulus, and these changes were greater for Know than for Remember responses. ERF signal changes linked to recollection were analyzed in a later epoch (500-800 ms), and were greater for Remember than for Know responses. The implications of the findings in the two epochs are discussed below.

\section{$300-500 \mathrm{~ms}$}

The results from a substantial number of ERP studies of memory retrieval point to the presence of a neural index of familiarity between 300 and $500 \mathrm{~ms}$ poststimulus (for review, see Mecklinger, 2000; Rugg and Curran, 2007). In addition, in an ERF study incorporating the same design used in prior ERP studies, Bridson et al. (2009) reported the presence of an effect that they linked with familiarity over left frontal scalp regions, where the effect was also located and analyzed in this experiment. Within the current experiment, moreover, further data consistent with the view that activity at sensors over left frontal scalp indexes familiarity comes from the analysis of the ERFs elicited by new judgments to studied words (misses). If familiarity is considered 
as a graded signal, then the average level of familiarity associated with misses should be lower than that associated with Know responses, but higher than that associated with correct rejections. Mean signal strengths for ERFs associated with misses were reliably lower than those associated with Know responses. Because signal strengths associated with correct rejections were used in the contrast that was used to identify the clusters for the key experiment analyses, a correct rejection versus miss comparison represents a biased contrast. Figure 1 does show, however, that signal strength for the miss response category lies between that for correct responses to old and to new test words.

The key finding in this epoch was that the index of familiarity was larger for Know than for Remember judgments. This outcome is predicted by a dual-process model in which the processes of recollection and familiarity are independent (Yonelinas, 1994, 2002), because the level of familiarity associated with all Know responses will exceed a criterion, whereas this is not the case for the level of familiarity associated with all Remember responses (Yu and Rugg, 2010). The result of averaging across multiple trials associated with the same behavioral outcomes will be a larger ERF index of familiarity associated with Know than with Remember responses.

Considerations about averaging across trials also lead to the prediction that, as the criterion for the level of familiarity sufficient to sanction a Know response becomes more liberal, the magnitude of an ERF old/new effect indexing familiarity will become more similar for items attracting Remember or Know responses. This prediction follows because an increasingly liberal criterion for familiarity-based responding means an increase in the proportion of the familiarity strength distribution from which familiarity strengths associated with Remember and Know responses are drawn (for comparable criterion placement arguments, see Azimian-Faridani and Wilding, 2006; Curran et al., 2007). A related prediction is that, when all or the vast majority of items in a task are sufficiently familiar to sanction a Know response, the magnitude of an index of familiarity will converge for Remember and for Know responses.

One outstanding question is why the direction of the differences between indices of familiarity for Remember and for Know responses reported here has not been documented in ERP studies where the same contrasts have been made and in which the probabilities of Remember and Know responses have been similar to those in this experiment (Düzel et al., 1997; Duarte et al., 2004; Woodruff et al., 2006; Yu and Rugg, 2010). The most common finding in these ERP studies is no reliable differences between the magnitudes of a familiarity index for items attracting Remember or Know responses. An appeal to the differential sensitivities of two distinct measures of neural activity is likely to be an important consideration when addressing this disparity, with conjoint acquisition of ERPs and ERFs offering a means of assessing directly whether, under precisely the same experimental circumstances, the sensitivities of the two modalities to the process of familiarity are comparable (Bridson et al., 2009).

The inclusion of misses for the key experiment contrasts here is a departure from approaches taken in the majority of ERP studies of memory retrieval operations, where response categories associated with correct judgments to studied test items are commonly compared against those associated with correct rejections. In this experiment, the use of the correct rejection signal strength measures in the analysis that was used to identify clusters from which key signal strength measures were extracted precluded the use of the category again in the subsequent paired contrasts. It can be argued, however, that neural activity associ- ated with misses has one advantage in this context over that associated with correct rejections, which is that neural activity elicited by studied items that does not contribute to explicit memory judgments should be represented approximately equally in response categories where old words are separated according to response accuracy.

\section{$500-800 \mathrm{~ms}$}

Neural activity linked to recollection has been identified in numerous ERP studies in this time period (Friedman and Johnson, 2000; Donaldson et al., 2003; Rugg, 2004), and the cluster at which the modulation of interest was analyzed in this experiment corresponds closely to the modulation linked previously to recollection by Bridson and colleagues (2009). In this experiment, mean signal strengths for words subjected to shallow encoding and attracting Remember responses at test were greater than those for misses, as well as words subjected to shallow encoding and given a Know response. Mean signal strengths for these final two categories did not differ. These outcomes are consistent with the view that this ERF old/new effect indexes recollection, which should not be associated to any substantive degree with misses or with studied words given Know responses.

This outcome is not in and of itself predicted uniquely by any one model described in the Introduction, but the differences between the profile of this effect across response categories and the effect described in the 300-500 ms epoch are consistent with the view that two functionally dissociable processes have been identified. The data can therefore be used to argue against the view that Remember and Know responses reflect differences in the degree of an undifferentiated strength signal (Donaldson, 1996). Moreover, the combination of one old/new effect that is larger for Know than for Remember responses, and a second for which the reverse is true, is at odds with the view that Remember responses are associated, on average, with higher levels of recollection as well as familiarity than are Know responses (Wixted, 2007, 2009).

\section{Conclusions}

In conclusion, the findings reported here are consistent with accounts in which recollection and familiarity make independent contributions to memory judgments (Yonelinas, 1994). They do not support accounts where Remember and Know responses reflect different degrees of memory strength. As a consequence, the findings constrain proposals about the roles played by distinct brain regions in human memory operations (Eichenbaum et al., 2007; Squire et al., 2007).

\section{References}

Azimian-Faridani N, Wilding EL (2006) The influence of criterion shifts on electrophysiological correlates of recognition memory. J Cogn Neurosci 18:1075-1086.

Berry CJ, Shanks DR, Speekenbrink M, Henson RN (2012) Models of recognition, repetition priming, and fluency: exploring a new framework. Psychol Rev 119:40-79.

Bridson NC, Muthukumaraswamy SD, Singh KD, Wilding EL (2009) Magnetoencephalographic correlates of processes supporting long-term memory judgments. Brain Res 1283:73-83.

Curran T (1999) The electrophysiology of incidental and intentional retrieval: ERP old/new effects in lexical decision and recognition memory. Neuropsychologia 37:771-785.

Curran T (2000) Brain potentials of recollection and familiarity. Mem Cognit 28:923-938.

Curran T, DeBuse C, Leynes PA (2007) Conflict and criterion setting in recognition memory. J Exp Psychol Learn Mem Cogn 33:2-17.

Donaldson DI, Wilding EL, Allan K (2003) Fractionating retrieval from episodic memory using event-related potentials. In: The cognitive neurosci- 
ence of memory: episodic encoding and retrieval (Parker AE, Wilding EL, Bussey TJ, eds.), pp 39-58. Hove: Psychology.

Donaldson W (1996) The role of decision processes in remembering and knowing. Mem Cognit 24:523-533.

Duarte A, Ranganath C, Winward L, Hayward D, Knight RT (2004) Dissociable neural correlates for familiarity and recollection during the encoding and retrieval of pictures. Brain Res Cogn Brain Res 18:255-272.

Düzel E, Yonelinas AP, Mangun GR, Heinze HJ, Tulving E (1997) Eventrelated brain potential correlates of two states of conscious awareness in memory. Proc Natl Acad Sci U S A 94:5973-5978.

Eichenbaum H, Yonelinas AP, Ranganath C (2007) The medial temporal lobe and recognition memory. Ann Rev Neurosci 30:123-152.

Friedman D, Johnson R Jr (2000) Event-related potential (ERP) studies of memory encoding and retrieval: a selective review. Microsc Res Tech $51: 6-28$.

Gardiner JM, Java RI (1993) Recognising and remembering. In: Theories of memory (Collins A, Conway MA, Gathercole SE, Morris PE, eds.), pp 163-188. Hillsdale, New Jersey: Erlbaum.

Greenhouse GW, Geisser S (1959) On methods in the analysis of profile data. Psychometrika 49:95-112.

Mecklinger A (2000) Interfacing mind and brain: a neurocognitive model of recognition memory. Psychophysiology 37:565-582.

Rajaram S (1993) Remembering and knowing: two means of access to the personal past. Mem Cognit 21:89-102.

Rugg MD (2004) Retrieval processing in human memory: electrophysiological and fMRI evidence. In: The new cognitive neurosciences (Gazzaniga MS, ed.), pp 727-737. Boston, Massachusetts: MIT.

Rugg MD, Curran T (2007) Event-related potentials and recognition memory. Trends Cogn Sci 11:251-257.
Squire LR, Wixted JT, Clark RE (2007) Recognition memory and the medial temporal lobe: a new perspective. Nat Rev Neurosci 8:872-883.

Tendolkar I, Rugg M, Fell J, Vogt H, Scholz M, Hinrichs H, Heinze HJ (2000) A magnetoencephalographic study of brain activity related to recognition memory in healthy young human subjects. Neurosci Lett 280:69-72.

Tulving E (1985) Memory and consciousness. Canadian Psychologist 26:1-12.

Vrba J, Robinson SE (2001) Signal processing in magnetoenceophalography. Methods 25:249-271.

Wixted JT (2007) Dual-process theory and signal-detection theory of recognition memory. Psychol Rev 114:152-176.

Wixted JT (2009) Remember/Know judgments in cognitive neuroscience: an illustration of the underrepresented point of view. Learn Mem 16:406-412.

Wixted JT, Mickes L (2010) A continuous dual-process model of remember/know judgments. Psychol Rev 117:1025-1054.

Woodruff CC, Hayama HR, Rugg MD (2006) Electrophysiological dissociation of the neural correlates of recollection and familiarity. Brain Res 1100:125-135.

Yonelinas AP (1994) Receiver-operating characteristics in recognition memory: evidence for a dual-process model. J Exp Psychol Learn Mem Cogn 20:1341-1354.

Yonelinas AP (2002) The nature of recollection and familiarity: a review of the 30 years of research. J Mem Lang 46:441-517.

Yonelinas AP, Parks CM (2007) Receiver operating characteristics in recognition memory: a review. Psychol Bull 133:800-832.

Yu SS, Rugg MD (2010) Dissociation of the electrophysiological correlates of familiarity strength and item repetition. Brain Res 1320:74-84. 